\title{
Dominance Weighted Social Choice Functions for Group Recommendations
}

\author{
A \\ Silvia ROSSI ${ }^{\mathrm{a}}$; Francesco BARILE ${ }^{\mathrm{b}}$; Antonio \\ $\mathrm{CASO}^{\mathrm{c}}$ \\ a Dipartimento di Ingegneria Elettrica e Tecnologie dell'Informazione, Universita' degli Studi di \\ Napoli “Federico II”, Napoli, Italy \\ b Dipartimento di Matematica e Applicazioni, Universita' degli Studi di Napoli “Federico II”, \\ Napoli, Italy \\ 'Dipartimento di Fisica, Universita' degli Studi di Napoli “Federico II”, Napoli, Italy
}

KEYWORD

Group Decision

Making; Social

Choice; Group

Recommendation;

Small Groups; Social

Networks

\section{ABSTRACT}

In travel domains, decision support systems provide support to tourists in the planning of their vacation. In particular, when the number of possible Points of Interest (POI) to visit is large, the system should help tourists providing recommendations on the POI that could be more interesting for them. Since traveling is, usually, an activity that involves small groups of people, the system should take simultaneously into account the preferences of each group's member. At the same time, it also should model possible intra-group relationships, which can have an impact in the group decision-making process. In this paper, we model this problem as a multi-agent aggregation of preferences by using weighted social choice functions, whereas such weights are automatically evaluated by analyzing the interactions of the group's members on Online Social Networks.

\section{Introduction}

We are involved in a Smart City project whose main mission is to develop ICT components with the aim of re-evaluating the cultural heritage fruition of the historic center of Naples. Some of the developed components aim at the creation of a framework, with web and mobile applications that helps tourists in the city. In this context, the goal of our research is to provide decision support systems to help tourists, as well as citizens, to effectively plan their activities by easily choosing attractions and local businesses to visit with the minimum effort. In particular, since the number of the available Points of Interest (POI) in the city is high, and since many tourists visit a city only for few days, it is not possible to visit and evaluate every POI. So the system should help the tourist to make a selection of what he/she believes to be the most valuable POI.

A decision support system like this has to take into account that, potentially, different group's members (not a single user) jointly select the activities to perform. It is necessary to provide a support to this group's decision-making process. In this case, it is necessary to suggest activities, or, more generally speaking, certain POI that maximize the group satisfaction, considering that the members' preferences can be different. In travel application domains, group profiles have been taken into account (Souffriau and Vansteenwegen, 2010), however, mainly as an optimization problem among POI. Moreover, in (Ardissono et al., 2003) intra-group

Regular Issue

Vol 4 n.1
Advances in Distributed Computing and Artificial Intelligence Journal 
relationships, such as children and the disabled were contemplated, while (McCarthy et al., 2006; Jameson, 2004) provided mechanism to help groups in deciding common attributes and features for their holidays.

Providing recommendation to groups is a challenging problem because of the diversity and the dynamics of intra-group relationships (Gartrell et al., 2010). It is widely recognized that one of the issues that has to be taken into account in the design and implementation of Group Decision Support Systems is the type of control over the group decision-making process (Jelassi and Foroughi, 1989). Hence, recommendation systems for groups need to capture both group members' preferences, but also key factors in the group decision process (Gartrell et al., 2010). For example, automatic voting/ranking mechanism often requires that all the agent involved have the same influence on the decision procedure, that is not the case in real group scenarios. There may be cases where the participants follow a democratic process in order to find a possible solution, and cases where the group is supported by a human leader or mediator. Real group interactions take into account intra-group roles and mutual influences. Moreover, the decision of a group member whether or not to accept a given recommendation may depend not only on his/her own evaluation of the content of the recommendation, but also on his/her beliefs about the evaluations of the other group members (Cantador and Castells, 2012). Users may be willing to accept last preferred activities in order to improve the group happiness.

We believe that such factors can be evaluated by analyzing the group interactions in Online Social Networks (OSNs). OSNs are widely recognized as effective ways to interact, communicate and collaborate with friends, but also to drive people's opinions. Moreover, OSNs interaction analysis can provide a viable way to obtain, without intruding the users with questionnaires, information about the social relationships and pattern of activities among the group's members. Some recent work has seen the emergence of a class of socially enhanced applications that leverage relationships from OSNs, especially to improve security and performance of network applications and online advertising (Wilson et al., 2009). While the attempt to infer meaningful relationships from social networks connectivity is often criticized from sociology researchers, the analysis of the interaction graphs in controlled situations (e.g., small and close groups) may provide useful insight. Finally, social networks analysis may lead to a misinterpretation of popularity as leadership that sometimes are highly correlated, but sometimes they are not. It was shown that cohesiveness of a group determines the correlation between these two concepts (Theodorson, 1957). Moreover, the cohesiveness of a group is a fundamental issue in facilitating the decision process. In a cohesive group, users self-needs can be sacrificed for the well-being of the whole group.

In this work, we show how social choice functions, typically used to merge agents' preferences, can be enhanced by using user weights automatically evaluated by an analysis of group members' relationships. In particular, our aim is to take concepts from research on users connectivity and on users communication activity in order to identify key users in OSNs. To validate our approach, we conducted two user studies with groups involved in a city trip planning. In the first, each user was asked to select his/her preferred POI, from a list, and then the group to provide a final decision. In the second, the users were asked to provide the rankings for the same POI and, then, to evaluate two solutions for the groups that are recommended by the system. Our results show that, even in the case of a simple extraction of the number of directed interactions, without a semantic analysis of the contents, it is possible to detect key users and implement "weighted" social choice functions highly accepted by the users.

Regular Issue

Vol 4 n.1

http://adcaij.usal.es
Advances in Distributed

Computing and Artificial Intelligence Journal

(c)Ediciones Universidad de Salamanca / CC B Y-NC-ND 


\section{Background and Related Works}

Recently, the problem of group recommendation has been addressed in the literature. Group recommendation approaches rely either on building a single user profile, resulting from the combination of users' profiles, or on merging the recommendation lists of individual users, at runtime, using different group decision strategies. The latter has been widely studied in Mathematics, Economics and Multi-agent systems (MAS), with the definition of Social Choice functions. These strategies, according to (Senot et al., 2010), can be classified as majority-based (mainly implemented as voting mechanisms to determine the most popular choices among alternatives), consensus-based (that try to average among all the possible choices and preferences), and role-based (that explicitly take into account possible roles and hierarchical relationships among members).

PolyLens (O'Connor et al., 2001) has been one of the first approaches to include social characteristics (such as the nature of a group, the rights of group members, and social value functions for groups) within the group recommendation process. It uses a Collaborative Filtering (CF) approach to produce recommendations for each user of the group, and a Least Misery (LM) strategy to aggregate these recommendations. Moreover, in (Ardissono et al., 2003) intra-group relationships, such as children and the disabled were contemplated; each group is subdivided into homogeneous subgroups of similar members that fit a stereotype, and recommendations are predicted for each subgroup and an overall preference is built considering some subgroups more influential.

The results presented in the literature showed that there is no strategy can be defined as the "best", but different approaches are better suited in different scenarios, depending from the characteristics of the specific group. Besides, traditional aggregation techniques do not seem to capture all the features of real-world scenarios. Many of these approaches do not consider the social relationships among group members at all (Gartrell et al., 2010). For example, automatic voting/ranking mechanisms often require that all the agents involved have the same influence on the decision procedure, while real group interactions take into account intra-group roles and mutual influences. Some members of the group could have a particular influence on the others, and the decision of a group member whether or not to accept a given recommendation may depend not only on his/her own evaluation of the content of the recommendation, but also on his/her beliefs about the evaluations of the other group members (Cantador and Castells, 2012). Following these ideas, in (Gartrell et al., 2010) Authors starts to evaluate the group members' weights, in terms of their importance or influence in a group, for movies recommendations. The scope of this work is similar to ours and it was tested on real groups. However, in (Gartrell et al., 2010) the defined group functions rely on the concept of "expertise" and "group dissimilarity", while the selection of the function is obtained by a "social value" derived from questionnaires. On the contrary, in our work, we argue that relevant parameters can be directly extracted, in a first approximation, by a "non-semantic" analysis of the interactions and by using an extension of widely used social choice functions. In (Gartrell et al., 2010), the Authors propose a dissimilarity measure that we used in our case studies, while an approach that provides group recommendations with explicit relationships within a family is proposed in (Berkovsky et al., 2009). Finally, approaches for large groups or small, but static, groups, as in (Vildjiounaite et al., 2008), are not applicable in our case since our groups do not have a history of common activities (as in (Seko et al., 2011)) and have to reach an agreement on a small set of items (i.e., on the activities to perform).

Consensus decision making has been explored, inspired by social networks, in (Salehi-Abari and Boutilier, 2014), where individual preferences are aggregated in a weighted social choice function that, as in our case,

Regular Issue

Vol 4 n. 1

http://adcaij.usal.es
Advances in Distributed Computing and Artificial Intelligence Journal 
takes into account local relationships with neighborhoods in a network. However, in (Salehi-Abari and Boutilier, 2014) the Authors do not specify how to evaluate such numerical relationships while they focus on computational aspects of scaling up with large networks of friends.

Finally, other classical MAS approaches tried to address the problem from a different perspective. In (Bekkerman et al., 2006) there is a negotiation agent for each group member. An individual recommendation system provides a recommendation for a set of items, and, in addition to this, an individual utility of each product for each user is evaluated, introducing a user preference model. The Negotiation protocol is different according to the cardinality of the group. A similar approach is used in (Garcia et al., 2009) and (Garcia and Sebastia, 2014). In this case, there are agents that act on behalf of group's members, and the recommendations are obtained as the result of the negotiation too. But, here, user agents are configurable in order to exhibit the desired behavior of the corresponding user. The negotiation model is a multi-party negotiation that centralize the communications through a negotiator agent, acting as mediator. It receives the proposals of the user agents, combines them into a single proposal, which is later broadcasted by the negotiator agent and analyzed by the user agents.

A different approach is used in (Carvalho and Macedo, 2013a), where the problem is modeled as a coalitional game. It assumes that the group can be divided into small subgroups, and it is possible to give a different recommendation to each subgroup, maximizing the social welfare function of the group. However, this approach is not applicable in cases whereas it is necessary to provide a recommendation for the whole group. In (Carvalho and Macedo, 2013b) is proposed an alternative approach, based on non-cooperative games. The problem is modeled as a game and the recommendation is determined by finding the Nash equilibrium of the game. This approach can be used in the case of great heterogeneity in the group.

\section{Material and Methods}

The aim of this work is to design a framework for group recommendation and decision support systems for touristic applications. Our first requirement was to build a system to provide personalized contents to users and that can work both with single users and with groups of users. Another issue was to be able to handle group memberships that may dynamically change in time. Here, as groups of friends, we refer to sets of people that meet, interact, or have some actual common bond in the physical world since they are planning real activities to perform together. Moreover, we assume groups that are willing to collaborate in order to maximize their social welfare. We decided to focus on merging the recommendation lists, generated for each individual user because it provides us with the flexibility required in the group formation process; single user's profiles and recommendations are built independently from the other group's members, and the users' recommendations are merged at the time of providing the group recommendations (e.g., only once the group is established). Finally, our system will not provide a single item recommendation, but sequences or sets, as required by the touristic application domain. The complete process, we aim to achieve, is depicted in Figure 1, where we have two separate processes in order to build users' profiles and recommendations, and a group consensus function to dynamically accounts for group needs, and which take into account relationships among members extracted from the interactions on a social network.

Formally, given a group of $n$ users $G=\{1, \ldots, n\}$ and a set of $m$ POI $P=\{1, \ldots, m\}$, each user $i \in G$ has a preference profile $\succ_{i}$ over $P\left(\succ_{i}=\left\{r_{i}(1), \ldots, r_{i}(m)\right\}\right)$, with $r_{i}(x) \in \mathcal{R}$, which represents the $i$ 's rank

Regular Issue

Vol 4 n.1

http://adcaij.usal.es
Advances in Distributed

Computing and Artificial Intelligence Journal 


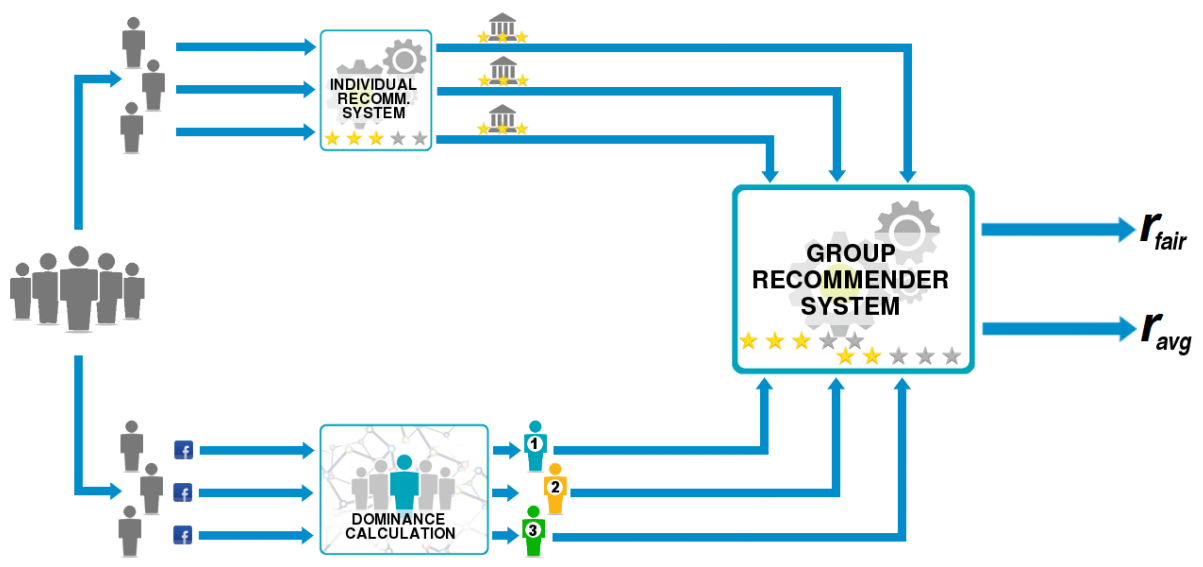

Figure 1: System Architecture for Single User and Group Recommendation.

for the $x$ POI, and it is, eventually, retrieved by some recommendation mechanism. The goal of the proposed work is to implement social choice functions $S C: \succ^{n} \rightarrow \succ_{G}$, that aggregates all the preferences profiles in $\succ_{G}=\left\{r_{G}(1), \ldots, r_{G}(m)\right\}$, that is the correspondent ranking as evaluated for the group. In this work, we suppose to have complete and available preference profiles (e.g., they are directly expressed by the users or provided by a recommendation system for single users). In future works such preferences will be extracted with the use of recommendation processes.

Differently from typical movie group recommendation, here the problem is not to select a single item for the group, but a subset of activities (top K) for close friends. Hence, there is room for potentially accommodate the needs of each group member. In our framework, a majority evaluation of activities may have the potential risk to leave some users dissatisfied, while, in our opinion, a close group will try to satisfy everyone needs, as long as possible. Finally, according to (Masthoff, 2011), users involved in real interaction seem to care about fairness and to avoid misery. For these reasons we decided to use a fairness strategy and one based on average satisfaction. Our idea is to weigh such functions with a measure of the influence of each user on the other group's members, and, consequently, on the group's final decision. To make this, we evaluate the weight of the relationship between pairs of users from the analysis of the interactions on an OSN.

In order to define our social choice functions, we analyze the possibility to evaluate, in a first approximation, the "role" of a specific relationship from the analysis of interactions on a social network, without the help of semantic features. In (Adali et al., 2012), the Authors showed that the analysis of only the user behaviors is practically equivalent, in terms of ability to determine the different types of relationships between pairs of individuals interacting in social media, to methods based on text analysis. Information exchanges between actors are dependent on social attachments that produce expectations of trust and reciprocity. In particular, in this work, we are interested in dominance and relationship-focused leadership or influence, from the analysis of small group members' interactions in OSNs. Many social networks analysis approaches assume binary, symmetric relationships of equal value between all directly connected users with the main focus in analyzing big networks, while, in our approach, we deal with small groups of friends that are, most of the time, totally connected one to the others. Moreover, differently from binary networks, in reality, an individual has relationships of varying quality (Banks and Wu, 2009; Gilbert and Karahalios, 2009a). However, OSNs

Regular Issue

Vol 4 n.1

http://adcaij.usal.es
Advances in Distributed Computing and Artificial Intelligence Journal 
keep tracks of the type of interaction among the users that can be used for modeling (Xiang et al., 2010) intra-user relationships.

There is a number of attempts to generalize the three node centrality measures to weighted networks (Newman, 2004; Opsahl et al., 2010). Here, To compute the users' ranking, we decided to use a simple "non-semantic" approach defined in our previous work (Caso and Rossi, 2014). For each user $i \in G$ we evaluate his/her dominance value, as the value $D(i) \in[0,1]$. Dominance values are computed by analyzing the popularity of each user within the group, and evaluating the number of directed interactions of each user towards the other group's members. Such popularity values are obtained implementing an extension of the well-known PageRank algorithm (Brin and Page, 1998) starting from the users' interactions on the social network facebook.com. For each user $i \in G$ we evaluate his/her dominance value, as the value $D(i) \in[0,1]$. Dominance values are computed by analyzing the popularity of each user within the group, and evaluating the number of directed interactions of each user towards the other group's members. Such popularity values are obtained implementing an extension of the well-known PageRank algorithm (Brin and Page, 1998) starting from the users' interactions on the social network facebook.com. As in the classic PageRank, each user inherits a portion of popularity from other users.

This ranking function is defined as follows:

$$
D(x)=\frac{1-d}{|F|}+d \sum_{i \in F} \frac{w(i, x)}{w(i)} D(i)
$$

where, $|F|$ is the total number of friends in the group and $\mathrm{d}$ (with $0 \leq d \leq 1$ ) is a dampening factor set to 0.85 (this value is often considered the default value for PageRank calculations (Langville and Meyer, 2004)). In the second part of Equation 1, the user $x$ inherits a portion of popularity from the other $i$ group's members. In detail, this proportion is calculated by considering both the $i$-th friend's popularity and the weight of the communication activity of the $i$-th friend towards the user $x(w(i, x))$, normalized with respect to the total communication activity of the $i$-th friend with all the members of the group $(w(i))$. The rationale of this choice is that the frequency of directed communication (or interaction) from the user $i$ towards the user $x$ is an index of the strengths of the directed tie $i-x$ (which can have a different value with respect to the tie $x-i$, and, hence, have a different impact on the evaluation of the $x$ 's popularity within the group).

Such weights are calculated by considering some of the communication activities between couple of users on the OSN Facebook.com, collecting a combination of data arising from (Gilbert and Karahalios, 2009b). Referring to the activity graph of friends' relationships, $w(i, x)$ evaluates the edges from the user $i$ to the user $x$, which represent the activities with $i$ as source and $x$ as receiver.

The dominance values are, then, used as weights into the two social choice strategies described in the next subsections. The architecture of our recommendation system is shown in Figure 1. We can see that single users' profiles are used to obtain the individuals' recommendations, while the information about the interactions on the social network is used to compute the Popularity (Dominance) rankings. Both these data are used from the Group Recommendation System to provide the final choices for the whole group using the two strategies.

Regular Issue

Vol 4 n.1

http://adcaij.usal.es
Advances in Distributed

Computing and Artificial Intelligence Journal 


\begin{tabular}{|c|c|c|c|c|c|c|c|c|c|}
\hline$x$ & 1 & 2 & 3 & 4 & 5 & 6 & 7 & 8 & Table 1: An example of $r_{\text {fair. }}$ Users are ordered from \\
\hline$r_{1}(x)$ & 1 & 5 & 3 & 1 & 2 & $\underline{\mathbf{5}}$ & 4 & & 3. The numbers in bo \\
\hline$r_{2}(x)$ & 3 & $\underline{4}$ & 1 & 2 & 5 & 3 & 2 & & of the user's $K$ preferred POI, while the ratin \\
\hline$r_{3}(x)$ & 1 & 3 & 2 & 5 & 1 & 4 & 3 & & POI that causes the least $n$ \\
\hline fair $(x)$ & 1 & r & 3 & 2 & $J$ & 0 & 4 & & orlin \\
\hline
\end{tabular}

\subsection{Fairness Strategy}

The idea behind this strategy $\left(r_{\text {fair }}\right)$ is trying to accommodate everyone in the group. A user can agree to perform activities that he/she does not like so much as long as he/she will be able to do something he/she likes with his/her friends. The strategy requires an ordering of the users. The first user's top $K$ choices are selected. Among them, the one that causes the least misery to the others is selected (in case of items with the same rating a non-deterministic choice is made), and the process is repeated with the successive user in the rank. The group POI ranking values are assigned in a descending order from $m$ to 1 . Finally, the group recommendation will correspond to the $K$ POI with the highest $r_{\text {fair }}$ values. An example of a possible application of this strategy is provided in Table 1.

One of the main issues with the use of this strategy is that, by changing the users' ordering, the selection process will produce a different result in the outcome, hence, we propose to use the $D(i)$ values to provide a ranking/to sort the users.

\subsection{Average Satisfaction Strategy}

As a second strategy, we developed an average satisfaction strategy $\left(r_{\text {avg }}\right)$. Inspired by (Gartrell et al., 2010), we defined a strategy that takes into account the dominance as a weight for the rating provided by the user (note that the sum of the dominance values in a group is equal to one). Moreover, a second factor, which can be considered in the evaluation, is a measure of dissimilarity among the users individual ratings. The proposed strategy to evaluate the group $G$ rating for the item $x$ is:

$$
r_{\text {avg }}(x)=\alpha \cdot \frac{1}{n} \sum_{i \in G}\left(D(i) \cdot r_{i}(x)\right)+\beta \cdot\left(1-\sigma_{r_{i}(x)}^{2}\right)
$$

where, $r_{i}(x)$ is the rate for the item $x$, made by the user $i, D(i)$ is evaluated according to (Caso and Rossi, 2014), and $\sigma_{r_{i}(x)}^{2}$ is the variance of $r_{i}(x)$ that accounts for the dissimilarity among the ratings of all the $i \in G$ for the item $x$. $\alpha$ and $\beta$ are weights. Once that the set $\succ_{\text {avg }}=\left\{r_{\text {avg }}(1), \ldots, r_{\text {avg }}(m)\right\}$ is obtained, this is normalized to get the final decision. The first $K$ activities $x$ with the highest $r_{\text {avg }}(x)$ values are selected. An example of a possible application of this strategy is provided in Table 2.

\section{Experiments and Evaluation}

To evaluate the proposed functions, we had to address the problem that, in our specific domain, there is no dataset that can be used for the evaluation. In fact, our application would require a dataset containing information about users interactions on a social network, information on the preferences of individual users,

Regular Issue

Vol 4 n.1

http://adcaij.usal.es
Advances in Distributed

Computing and Artificial Intelligence Journal 


\begin{tabular}{|c|c|c|c|c|c|c|c|c|c|}
\hline$x$ & 1 & 2 & 3 & 4 & 5 & 6 & 7 & 8 & Table 2: An example of the group \\
\hline$r_{1}(x)$ & 1 & 5 & 3 & 1 & 2 & 5 & 4 & 3 & choice selection using a averag \\
\hline$r_{2}(x)$ & 3 & 4 & 1 & 2 & 5 & 3 & 2 & 4 & satisfaction evaluation. \\
\hline$r_{3}(x)$ & 1 & 3 & 2 & 5 & 1 & 4 & 3 & 2 & considered leadership values are \\
\hline$r_{\text {avg }}(x)$ & 0.51 & 1.21 & 0.61 & 0.16 & 0.45 & 1.14 & 0.88 & 0.94 & $L_{1}=0.44, L_{2}=0.41$, and $L_{3}$ \\
\hline$\succ_{\text {avg }}$ & 3 & 8 & 4 & 1 & 2 & 7 & 5 & 6 & \\
\hline
\end{tabular}

and information on the final choices of the groups, in order to apply the proposed techniques and compare the obtained results. A dataset like this does not exist, so we decided to conduce two pilot studies with real users involved in the task of planning a trip in a city. In the first case, the aim is to evaluate the benefits of the introduction of the dominance values as weights or to order users in the proposed functions. In the second, we focus on the users' satisfaction with respect to the recommendations proposed by the system.

\subsection{A User Study with Binary Decisions}

In the first case study, we evaluated the behavior of 14 groups composed, in the average, of 3.36 close friends. 46 users took part in the experimentation ( 26 men and 20 women). The average age was 27.3 with a graduate education.

We asked each user to register on a specific web site using the credentials of facebook.com. Once registered, they were asked to imagine to plan a one-day visit in a specific city and to select three activities (from a checklist of ten items) and two restaurants (from a check list of eight) for the day. Since we do not want the users to be involved in strategic reasoning, we did not ask the users to express numerical ratings for the selected choices in this first setting. Hence, we will assign $r_{i}(x)=1$ if the user $i$ selects the POI $x$, and $r_{i}(x)=0$ otherwise. A screen-shot of the interface used to select the activities to perform is shown in Figure 2. The group was, then, asked to discuss, face-to-face, in order to obtain a shared and unique decision for the group. This final decision corresponds to the set $\succ_{G T}$ used to evaluate our functions.

In order to evaluate our results, we adapted the proposed fairness strategy to binary selections. Since a single vote is associated with each POI ( 0 or 1$)$, at each iteration a user (selected according to his/her dominance value in a descending order) proposes its first $K$ choices (with $K=3$ ). For each of the $K$ proposals the votes made by the other users are summed, and the choice with the higher sum is selected. Note that if $K$ is equal to the number of possible outcomes (as in this case), an activity, selected by all the member of the group, will be selected in the final decision. Finally, in order to evaluate the impact of the users' dominance, we also implemented a standard fairness version with a random users ordering $\left(r_{\text {st.fair }}\right)$.

As second strategy, we developed the weighted average satisfaction strategy. With binary choices, the evaluation of dissimilarity does not have a relevant impact on the final decision. Hence, the weights associated to Eq. 2 are $\alpha=1$ and $\beta=0$. As in the previous case, we also implemented the standard version of a simple averaging function $\left(r_{\text {st.avg }}\right)$.

We evaluated the similarity of the recommendation provided using the weighted version of the fairness function $\left(\succ_{\text {fair }}\right)$, average satisfaction function $\left(\succ_{\text {avg }}\right)$, and the standard implementation of such functions (i.e., $\succ_{\text {st.fair }}$ and $\succ_{\text {st.avg }}$ ) with respect to the groups' ground truth $\left(\succ_{G T}\right)$.

Aggregated results are reported in Table 3. With respect to their standard implementations, functions

Regular Issue

Vol 4 n.1

http://adcaij.usal.es
Advances in Distributed

Computing and Artificial Intelligence Journal 


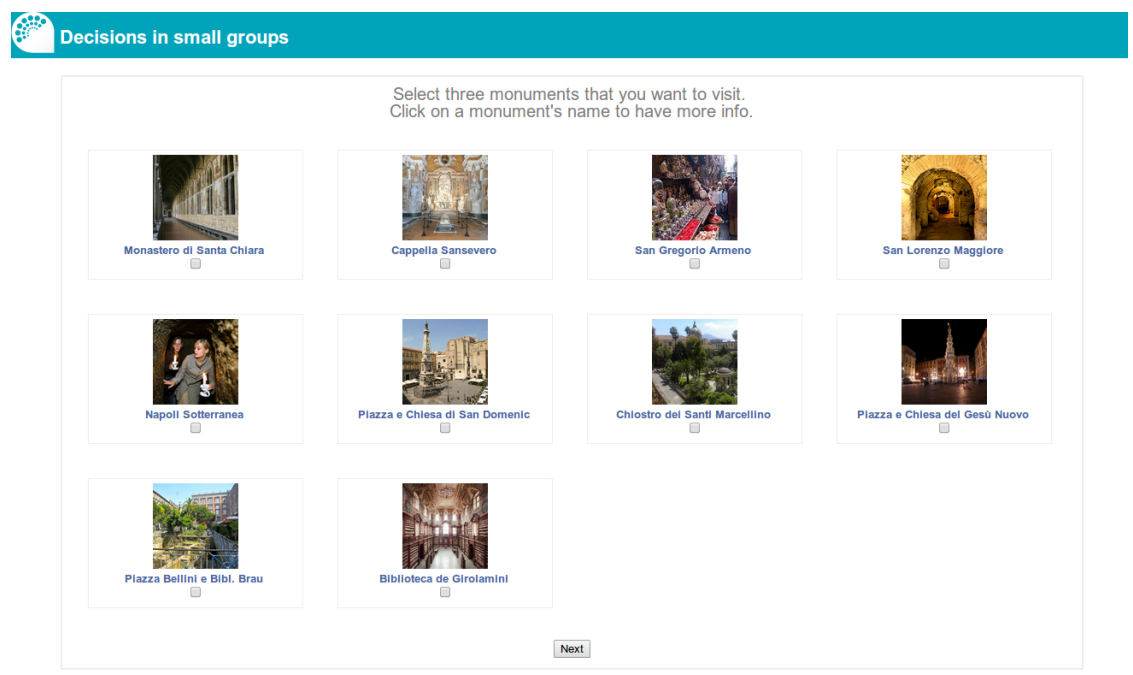

Figure 2: A screen-shot of the web page used to select the activities to perform.

\begin{tabular}{c||c|c||c|c} 
Similarity $\%$ & $\succ_{\text {st.fair }}$ & $\succ_{\text {fair }}$ & $\succ_{\text {st.avg }}$ & $\succ_{\text {avg }}$ \\
\hline$\succ_{G T}$ & $61 \pm 20$ & $66 \pm 18$ & $64 \pm 16$ & $74 \pm 12$
\end{tabular}

Table 3: Rate of similairty for $\succ_{\text {st.fair }}, \succ_{\text {fair }}, \succ_{\text {st.avg }}$ and $\succ_{\text {avg }}$ with respect to $\succ_{G T}$.

that take into account social relationships perform slightly better $(66 \%$ w.r.t $61 \%$ for fairness, and $74 \%$ w.r.t. $64 \%$ for average). A bigger improvement was noted for $r_{a v g}$. On the average, the $r_{\text {avg }}$ social choice function performs better than the $r_{\text {fair }}$, often guessing 4 on 5 activities. In many cases the responses of both strategies were the same, and there is only one case in which $r_{f a i r}$ performs better than $r_{a v g}$. Fairness strategy, in this simple binary case, with no information about rankings, suffers more of random choices made by the function in the case of activities with the same final score.

\subsection{A User Study with Rankings}

In this second case study, we evaluated the behavior of 17 new groups of friends composed, in the average, of 3.1 people. 53 users took part in the experimentation ( 26 men and 27 women). The average age was 26.8 with a graduate education.

This experiment was divided into two phases. In the first, as in the previous user study, each person was asked to register on a specific web site using the credentials of facebook.com. Once registered, he/she was asked to imagine planning a one-day visit in a specific city, but this time also to provide ratings (from one to five stars) to the ten proposed activities for the day and to the eight restaurants. Each rate corresponds to how likely it would be for the user to visit such place (or to eat in). The interface for the rating process is shown in Figure 3. For each activity/restaurant, a picture and a short description was provided to the users, plus a link to tripadvisor.com that provides additional information and a way to evaluate the popularity of the POI. Once that all the member of a group completed this first phase, we separately invited each member to login again and to complete the process by evaluating the proposed recommendations. Since the first phase was more

Regular Issue

Vol 4 n.1

http://adcaij.usal.es
Advances in Distributed

Computing and Artificial Intelligence Journal 
time consuming with respect to the previous user study, in the second phase users were only asked to evaluate the proposed results.

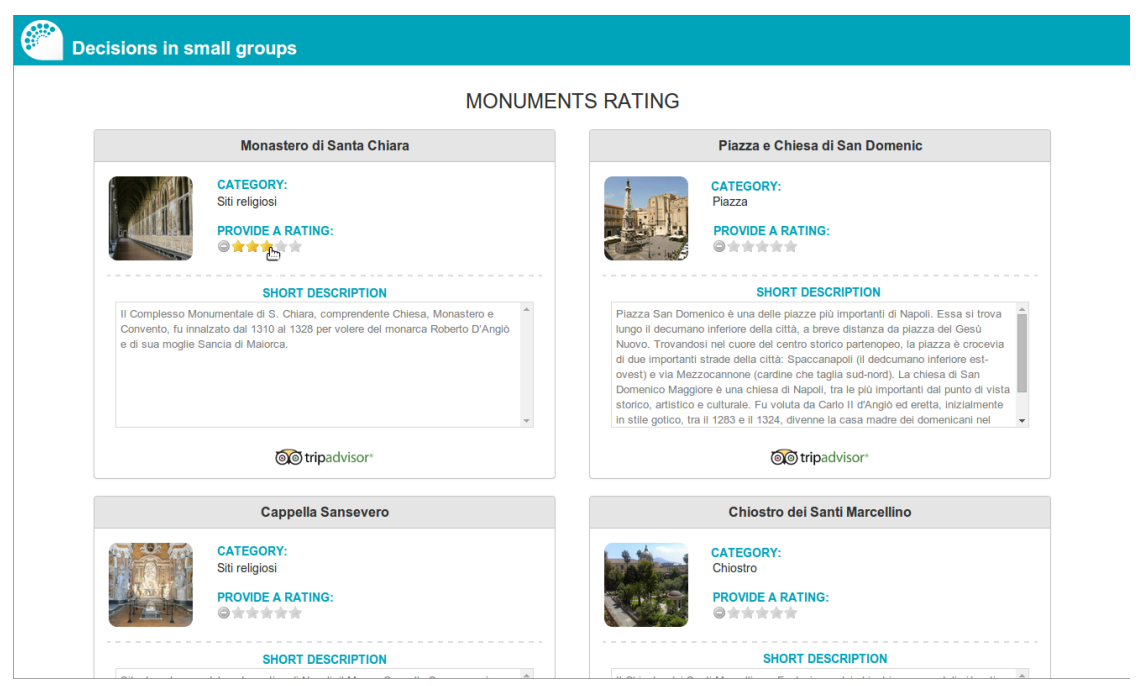

Figure 3: The rating page where participants were asked to rate activities.

In the $r_{\text {fair }}$ strategy the users were selected according to their dominance values in a descending order, with $K=3$. In the $r_{\text {avg }}$ strategy the weights associated to Equation 2 were $w_{1}=0.8$ and $w_{2}=0.2$ as suggested in (Gartrell et al., 2010).

Users were presented with both the recommendation provided by using the $r_{\text {fair }}$ and $r_{\text {avg }}$ functions (as in the previous case the top five activities were recommended). Moreover, the associated ratings for the proposed activities provided by all the members of the group in the first phase were shown. A screenshot of this interface is shown in Figure 4.

Finally, each user was requested to answer the following questions:

1. Which of the two proposed itineraries do you prefer? [None/First/Second/Both]

2. How do you rate the first itinerary? [1 to 5]

3. How do you rate the second itinerary? [1 to 5]

4. How much have you been influenced in the evaluations by the knowledge of your friends' ratings? [1 to 5]

In the average, the two used strategies differ one from the other for 2 choices, with some cases in which they provided the same results. In Table $4 \mathrm{a}$, we reported the acceptance rate mean percentage. In the average, two out of three members of each group accepted the proposed solution evaluated by the $r_{\text {fair }}$, while for $r_{\text {avg }}$ average value is a little bit smaller. Considering both the proposed options the system had an acceptance rate of $81 \%$. Moreover, considering directly all the users involved in the test, and not averaging on groups, 49 people out of 53 (i.e., $93 \%$ of the users) accepted at least one of the proposed itinerary.

In Table $4 \mathrm{~b}$, we reported the mean values for the users' ratings of the $\succ_{\text {fair }}$ proposed itinerary (i.e., question number 2), and $\succ_{\text {avg }}$ proposed itinerary (i.e., question number 3). Both the proposed results got, on

Regular Issue

Vol 4 n.1

http://adcaij.usal.es
Advances in Distributed

Computing and Artificial Intelligence Journal 


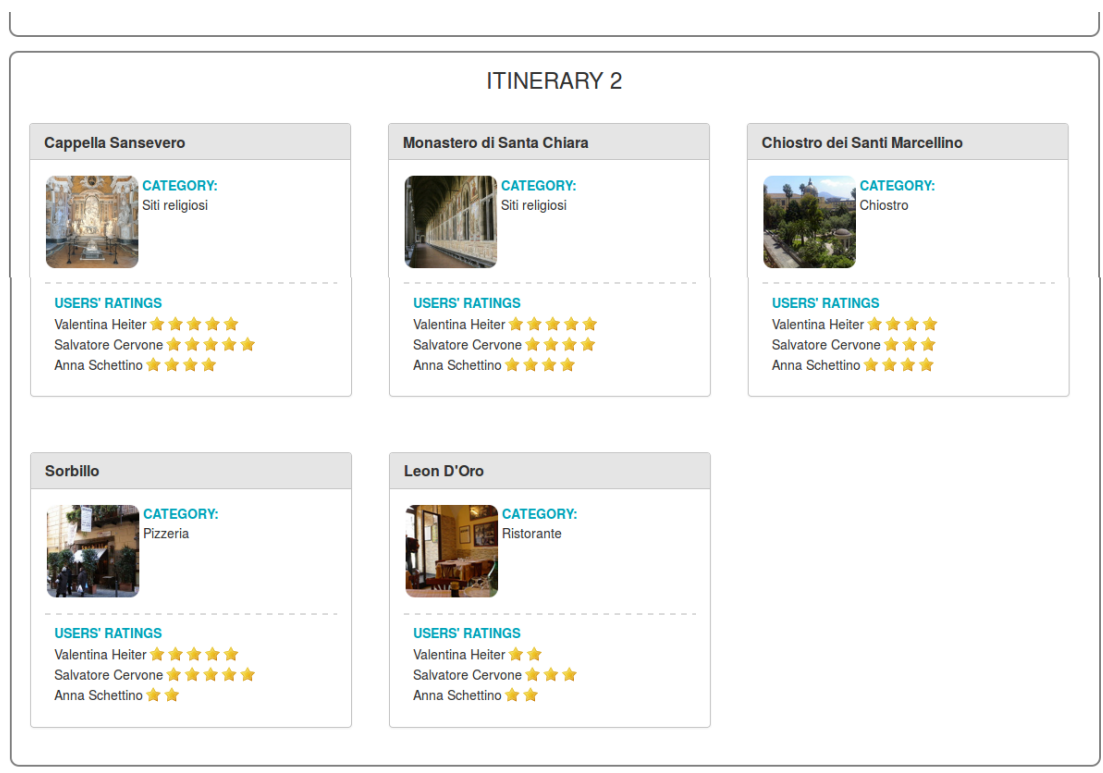

EVALUATE THE PROPOSED ITINERARIES

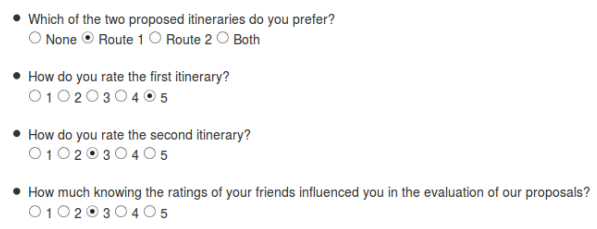

Figure 4: Web interface for accepting/rejecting results.

average, a good appreciation by the users (more than 4 stars). Moreover, we evaluated the Pearson correlation index $\rho$ between such ratings and the acceptance rates of the proposed strategies. As we expected, there is a strong correlation between the acceptance rate of the proposed strategies and their evaluations made by the users. The obtained value for $\succ_{\text {fair }}$ is $\rho=0.67$ (with a significance $p=0.003$ ), while for $\succ_{\text {avg }}$ is $\rho=0.77$ (with $p=0.0003$ ).

Surprisingly, the average evaluation of the impact of the friends' opinions on the evaluation of results ( friends in Table 4b) is 2.6. We imagine that such value is an effect of the testing procedure that ends with a private evaluation of the proposed solutions for the group.

\section{Conclusion}

The widespread use of social networks is modifying our way to live our cities and to plan/decide our activities. In particular, the way we plan a trip, and selects the places to visit or choose a restaurant are influenced by the information we can access on social media. Moreover, the online social networks (OSNs) can provide a viable way to obtain, without intruding the users with questionnaires, information about the users and their social relationships within communities and groups. While our long-term goal is to build a system that provides personalized contents to users in the touristic domain and that can work both with single users and

Regular Issue

Vol 4 n.1

http://adcaij.usal.es
Advances in Distributed

Computing and Artificial Intelligence Journal 


\begin{tabular}{l||c|c|c} 
Accept. \% & $\succ_{\text {fair }}$ & $\succ_{\text {avg }}$ & $\succ_{\text {fair }}+\succ_{\text {avg }}$ \\
\hline average & $66 \pm 32$ & $62 \pm 30$ & $81 \pm 20$ \\
(a) Acceptance rate for the $\succ_{\text {fair }}, \succ_{\text {avg }}$ and globally $\succ_{\text {fair }}$ \\
$+\succ_{\text {avg. }}$ \\
Users' rates & $\succ_{\text {fair }}$ & $\succ_{\text {avg }}$ & friends \\
\hline average & $4.3 \pm 0.6$ & $4.2 \pm 0.5$ & $2.6 \pm 0.6$
\end{tabular}

(b) Average ratings for the $\succ_{\text {fair }}, \succ_{\text {avg }}$ and for the evaluation of the friends' influence.

Table 4: Aggregate results for the second user study.

with groups of users, here, we focused on group recommendations. Providing recommendation to groups is a challenging problem because of the diversity of inter-group relationships and dynamics or their memberships.

In this paper, we propose a way to extract information about social relationships between group's members from the analysis of their activity on the OSN facebook.com, and how to use these information to evaluate a dominance factor that estimates the influence of each member on the group's final choice. Than we use these dominance values in the aggregation of preferences process, by defining two weighted social-choice functions. To validate our approach we conducted two pilot study, where we compare the recommendations provided by our weighted functions with their standard implementations. From these first analysis we can observe that our the weighted functions show better performances; in the first user study with binary selections, a bigger improvement was noted for the average satisfaction function, where the fairness seems to suffer more of random choices. On the contrary, in the second one, which involved POI ranking, fairness strategy has a bigger acceptance rate and appreciation evaluation.

A more deep analysis is necessary, involving greater number of users and possibly POI to choice in the pilot studies, to evaluate the scalability of the approach. Anyway, providing a group solution is not the last step in group recommendation since, when the recommendation involves a set of activities, the group may be involved in a subsequent interaction in order to take the final decision. Of course, the better is the recommendation, the easier is for the group to reach a consensus. In this direction, our next step will be to provide the group with interfaces to reach this final decision.

\section{Acknowledgments}

The research leading to these results has received funding from the Italian Ministry of University and Research and EU under the PON OR.C.HE.S.T.R.A. project (ORganization of Cultural HEritage for Smart Tourism and Real-time Accessibility).

\section{References}

Adali, S., Sisenda, F., and Magdon-Ismail, M., 2012. Actions Speak As Loud As Words: Predicting Relationships from Social Behavior Data. In Proc. of the 21st International Conference on World Wide

Regular Issue

Vol 4 n.1

http://adcaij.usal.es
Advances in Distributed

Computing and Artificial Intelligence Journal 
Web, pages 689-698. ACM.

Ardissono, L., Goy, A., Petrone, G., Segnan, M., and Torasso, P., 2003. INTRIGUE: Personalized recommendation of tourist attractions for desktop and handset devices. Applied Artificial Intelligence, 17(8):687-714.

Banks, L. and Wu, S., 2009. All Friends Are Not Created Equal: An Interaction Intensity Based Approach to Privacy in Online Social Networks. In International Conference on Computational Science and Engineering, volume 4, pages 970-974.

Bekkerman, P., Kraus, S., and Ricci, F., 2006. Applying cooperative negotiation methodology to group recommendation problem. In Proceedings of Workshop on Recommender Systems in 17th European Conference on Artificial Intelligence (ECAI 2006), pages 72-75. Citeseer.

Berkovsky, S., Freyne, J., and Coombe, M., 2009. Aggregation Trade Offs in Family Based Recommendations. In Nicholson, A. and Li, X., editors, Advances in Artificial Intelligence, volume 5866 of Lecture Notes in Computer Science, pages 646-655. Springer Berlin Heidelberg.

Brin, S. and Page, L., 1998. The Anatomy of a Large-scale Hypertextual Web Search Engine. Comput. Netw. ISDN Syst., 30(1-7):107-117.

Cantador, I. and Castells, P., 2012. Group Recommender Systems: New Perspectives in the Social Web. In Recommender Systems for the Social Web, volume 32 of Intelligent Systems Reference Library, pages 139-157. Springer.

Carvalho, L. A. M. and Macedo, H. T., 2013a. Generation of Coalition Structures to Provide Proper Groups' Formation in Group Recommender Systems. In Proceedings of the 22Nd International Conference on World Wide Web Companion, WWW' 13 Companion, pages 945-950. International World Wide Web Conferences Steering Committee.

Carvalho, L. A. M. C. and Macedo, H. T., 2013b. Users' Satisfaction in Recommendation Systems for Groups: An Approach Based on Noncooperative Games. In Proc. of the 22Nd Int. Conf. on World Wide Web Companion, WWW' 13 Companion, pages 951-958.

Caso, A. and Rossi, S., 2014. Users Ranking in Online Social Networks to Support POIs Selection in Small Groups. In Extended Proceedings of the 22nd Conference on User Modelling, Adaptation and Peronalization - UMAP 2014, pages 5-8.

Garcia, I. and Sebastia, L., 2014. A negotiation framework for heterogeneous group recommendation. Expert Systems with Applications, 41(4):1245-1261.

Garcia, I., Sebastia, L., and Onaindia, E., 2009. A Negotiation Approach for Group Recommendation. In Proceedings of the 2009 International Conference on Artificial Intelligence, Las Vegas Nevada, USA, pages 919-925. CSREA Press.

Gartrell, M., Xing, X., Lv, Q., Beach, A., Han, R., Mishra, S., and Seada, K., 2010. Enhancing Group Recommendation by Incorporating Social Relationship Interactions. In Proc. of the 16th ACM International Conference on Supporting Group Work, pages 97-106. ACM.

Gilbert, E. and Karahalios, K., 2009a. Predicting Tie Strength with Social Media. In Proceedings of the SIGCHI Conference on Human Factors in Computing Systems, CHI '09, pages 211-220. ACM.

Gilbert, E. and Karahalios, K., 2009b. Predicting Tie Strength with Social Media. In Proceedings of the SIGCHI Conference on Human Factors in Computing Systems, CHI '09, pages 211-220. ACM, New York, NY, USA.

Jameson, A., 2004. More Than the Sum of Its Members: Challenges for Group Recommender Systems. In

Regular Issue

Vol 4 n.1

http://adcaij.usal.es
Advances in Distributed

Computing and Artificial Intelligence Journal 
Proceedings of the Working Conference on Advanced Visual Interfaces, AVI '04, pages 48-54. ACM.

Jelassi, M. T. and Foroughi, A., 1989. Negotiation support systems: an overview of design issues and existing software. Decision Support Systems, 5(2):167 - 181.

Langville, A. N. and Meyer, C. D., 2004. Deeper inside pagerank. Internet Mathematics, 1:2004.

Masthoff, J., 2011. Group Recommender Systems: Combining Individual Models. In Recommender Systems Handbook, pages 677-702. Springer US.

McCarthy, K., McGinty, L., Smyth, B., and SalamÃş, M., 2006. The Needs of the Many: A Case-Based Group Recommender System. In Roth-Berghofer, T., GÃúker, M., and GÃijvenir, H., editors, Advances in Case-Based Reasoning, volume 4106 of Lecture Notes in Computer Science, pages 196-210. Springer Berlin Heidelberg.

Newman, M. E. J., 2004. Analysis of weighted networks. Phys. Rev. E, 70.

O'Connor, M., Cosley, D., Konstan, J. A., and Riedl, J., 2001. PolyLens: A Recommender System for Groups of Users. In Proc. of the 7th European Conf. on CSCW, pages 199-218.

Opsahl, T., Agneessens, F., and Skvoretz, J., 2010. Node centrality in weighted networks: Generalizing degree and shortest paths. Social Networks, 32(3):245 - 251.

Salehi-Abari, A. and Boutilier, C., 2014. Empathetic Social Choice on Social Networks. In 13th International Conference on Autonomous Agents and Multiagent Systems, pages 693-700.

Seko, S., Yagi, T., Motegi, M., and Muto, S., 2011. Group Recommendation Using Feature Space Representing Behavioral Tendency and Power Balance Among Members. In Proc. of the Fifth ACM Conference on Recommender Systems, pages 101-108.

Senot, C., Kostadinov, D., Bouzid, M., Picault, J., Aghasaryan, A., and Bernier, C., 2010. Analysis of Strategies for Building Group Profiles. In User Modeling, Adaptation, and Personalization, volume 6075 of $L N C S$, pages $40-51$.

Souffriau, W. and Vansteenwegen, P., 2010. Tourist Trip Planning Functionalities: State-of-the-Art and Future. In Current Trends in Web Engineering, volume 6385 of LNCS, pages 474-485.

Theodorson, G. A., 1957. The Relationship between Leadership and Popularity Roles in Small Groups. American Sociological Review, 22(1):pp. 58-67.

Vildjiounaite, E., Kyll onen, V., Hannula, T., and Alahuhta, P., 2008. Unobtrusive Dynamic Modelling of TV Program Preferences in a Household. In Changing Television Environments, volume 5066 of LNCS, pages $82-91$.

Wilson, C., Boe, B., Sala, A., Puttaswamy, K. P., and Zhao, B. Y., 2009. User Interactions in Social Networks and Their Implications. In Proceedings of the 4th ACM European Conference on Computer Systems, pages 205-218. ACM.

Xiang, R., Neville, J., and Rogati, M., 2010. Modeling Relationship Strength in Online Social Networks. In Proceedings of the 19th International Conference on World Wide Web, WWW '10, pages 981-990. ACM.

Regular Issue

Vol 4 n.1

http://adcaij.usal.es
Advances in Distributed

Computing and Artificial Intelligence Journal 


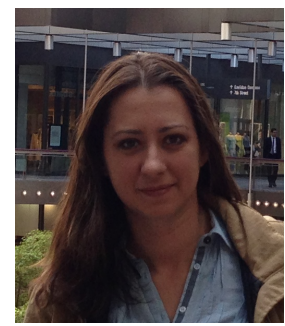

Silvia Rossi is currently assistant professor at the University of Naples "Federico II" (department of Electrical Engineering and Information Technologies). She received the M.Sc. degree in Physics from University of Naples "Federico II", Italy, in 2001, and the Ph.D. in "Information and Communication Technologies" from University of Trento, Italy, in 2006. Her research interests include Artificial Intelligence, Multi-agent System, Cognitive Robotics and Human-Robot Interaction.

e-mail: silvia.rossi@unina.it

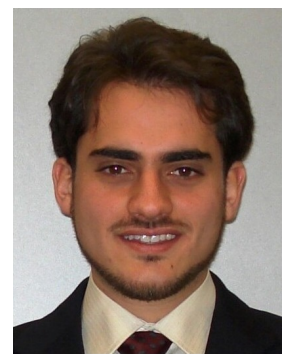

Francesco Barile is a Ph.D. Student in Computer Science at the University of Naples "Federico II", department of Mathematics and Applications. He achieved a Master degree in Computer Science at the University of Naples "Federico II", Italy, in 2012. His research interests include Multi-agent System, Recommendation Systems and Social Network Analysis.

e-mail: francesco.barile@unina.it

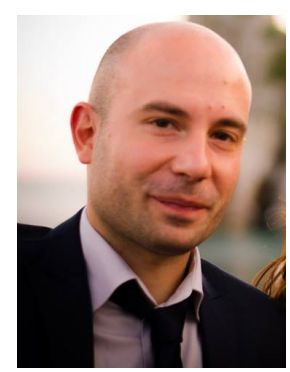

Antonio Caso was born on February 5th, 1984 in Naples (NA, Italy). He obtained a Master degree in Computer Science at University of Naples "Federico II", Italy, in 2013, with a thesis on Multimodal Interaction and Anger Detection Mechanism based on speech. In the last two years, he worked in the OR.C.HE.S.T.R.A. project, focusing his research on recommender systems for touristic systems and advanced web development.

e-mail: antonio.caso@unina.it

Regular Issue

Vol 4 n.1

http://adcaij.usal.es
Advances in Distributed

Computing and Artificial Intelligence Journal 